\title{
Electro Paramagnetic Resonance Studies to Determine the Dopant Site Occupancy of Dy-Sm doped Magnesium Ferrite for Micro Strip Patch Antenna Substrate
}

\author{
Vasant Naidu \\ Professor/ECE \\ Sethu Inst. of Tech., \\ Pulloor - 626115, \\ Tamilnadu, India
}

\author{
S. Gayathri Devi \\ Asst Professor/ECE \\ Sethu Inst. of Tech., \\ Pulloor - 626115, \\ Tamilnadu, India
}

\author{
R. Legadevi \\ Research Asst \\ Sethu Inst. of Tech., \\ Pulloor - 626115, \\ Tamilnadu, India
}

\author{
Lakshmi Priya \\ Asst Professor/ECE \\ Sethu Inst. of Tech., \\ Pulloor - 626115, \\ Tamilnadu, India
}

\begin{abstract}
In the present study, site occupancy of Dysprosium (Dy), Samarium $(\mathrm{Sm})$ doped Magnesium ferrite were investigated by electro paramagnetic resonance (EPR). Point charge calculations were used to predict the EPR spectrum of each lanthanide in A- and B-sites. Different EPR spectra are expected for A- versus B-site substitution when $\mathrm{Sm}^{3+}$ and $\mathrm{Dy}^{3+}$ are the dopants. The experimentally observed doping behavior of $\mathrm{Dy}^{3+}-\mathrm{Sm}^{3+}$ in $\mathrm{MgFe}_{2} \mathrm{O}_{4}$ suggests that as a $\mathrm{Sm}^{3+}$ cation it is on the A-site. EPR signal intensity suggested the amphoteric behavior due to $\mathrm{Dy}^{3+}$ in the magnesium ferrite material as $\mathrm{Dy}^{3+}$ was found to be a B-site dopant. These studies will be helpful for the analytical studies of permittivity and permeability to design Microstrip patch antenna to be used in pervasive computing.
\end{abstract}

Keywords: EPR, Dy-Sm Doped Magnesium ferrite, Dopant site.

\section{INTRODUCTION}

In an interesting review [1], electron paramagnetic resonance (EPR) is listed among the less developed methods for quantitative determination of site occupancies. However, for most transition metals and for rare earth ions that are present only in small amounts (up to about $1 \%$ per site), it still is the most powerful method. That is because this method gives unambiguous information about the valence state and site symmetry, and, at least for some ion.

\begin{abstract}
Magnesium ferrite, doped with lanthanide (Dy- Sm) ions, has found its use as a multilayer dielectric substrate [2]. It was observed that, certain lanthanides, such as Ho, Dy, Sm and sometimes Er, can improve the resistance of the magnesium ferrite due to electrochemical or time dependent failure, when fired in low oxygen partial pressures[3]. It is already mentioned that "magic" dopants can choose their site occupancy as a result of the local $\mathrm{Mg} / \mathrm{Fe}$ ratio and oxygen partial pressure during firing[4] and since these dopants were also termed "amphoteric" due to the site change of a well defined valence dopant and causing a change of the relative charge[5].
\end{abstract}

Here EPR studies are used to investigate a series of lanthanide-doped magnesium ferrite, with the chief aim for determining the site occupancy of these lanthanides. To facilitate the detection of amphotericity, these studies for the samples that are either magnesium-rich or ferrite-rich $(\mathrm{Mg} / \mathrm{Fe})$ 1.01 or 0.99 , respectively). The Mg-rich samples will drive amphoteric dopants to more frequently occupy B-sites, where as Fe-rich samples will drive such dopants more frequently into the A-sites. To determine the cubic crystalline electric field parameters for both A- and B-site locations for each particular lanthanide dopant, the analytical calculations for the point charge model is being considered Prediction of the fundamental lowest energy level of the $3^{+}$lanthanide in both types of site becomes possible. This then allows to assess whether A- or B-site dopant locations will have different EPR spectra [6].

\section{THEORY, THEORETICAL RESULTS,}

\section{AND DISCUSSION}

It is seen that the interaction with the crystalline electric field is much weaker than the spin-orbit coupling for the lanthanide ions [7]. There a sum of all interactions is expressed as a Hamiltonian

$$
H=H_{f i}+H_{s o}+H_{c f}+H_{s}+H_{h f}
$$

where $\mathrm{H}_{\mathrm{fi}}+\mathrm{H}_{\mathrm{so}} \gg \mathrm{H}_{\mathrm{cf}} \gg \mathrm{H}_{\mathrm{z}}+\mathrm{H}_{\mathrm{hf}}$,

$\mathrm{H}_{\mathrm{fi}}$ is the Hamiltonianof the free ion, Hso is the spin-orbit Hamiltonian, Hcf is the crystal field Hamiltonian, $\mathrm{H}_{\mathrm{z}}$ is the Zeeman Hamiltonian, and $\mathrm{H}_{\mathrm{hf}}$ is the hyperfine Hamiltonian.

The action of the crystal field upon the free ion can partially remove the degeneracy from among the various $\Gamma_{i}$ states.Therefore the crystalline electric field is a perturbation on the $2 \mathrm{~J}+1$ degeneracy of the free-ion ground state. Here $C 4, k$ and $C 6, k$ are constants, unique to each type of coordination sphere; $\langle r 4\rangle$ and $\langle r 6\rangle$ are the fourth- and sixthorder ionic radii; $r^{k}$ is the radius of the coordination sphere (distance from the dopant to an ion in the sphere ) $Z_{k}$ is the charge of an ion in the coordination sphere. The terms b4 and b6 can then be found by summing b4,k and b6,k over all possible shells. Because we are performing these calculations for two different lanthanides, it is useful to factor out all the lanthanide-specific parameters that do not change as a function of $\mathrm{k}$. This yields where and where From the equations set forth by Bureau et al.,[8] it can be shown that the constants $\mathrm{C} 4, \mathrm{k}$ and $\mathrm{C} 6, \mathrm{k}$ can be evaluated according to the following expressions and where $\mathrm{N}$ is the total number of ions, i, in each shell, $\mathrm{k}$, and Yl m(õi,_i) is a spherical harmonic. 


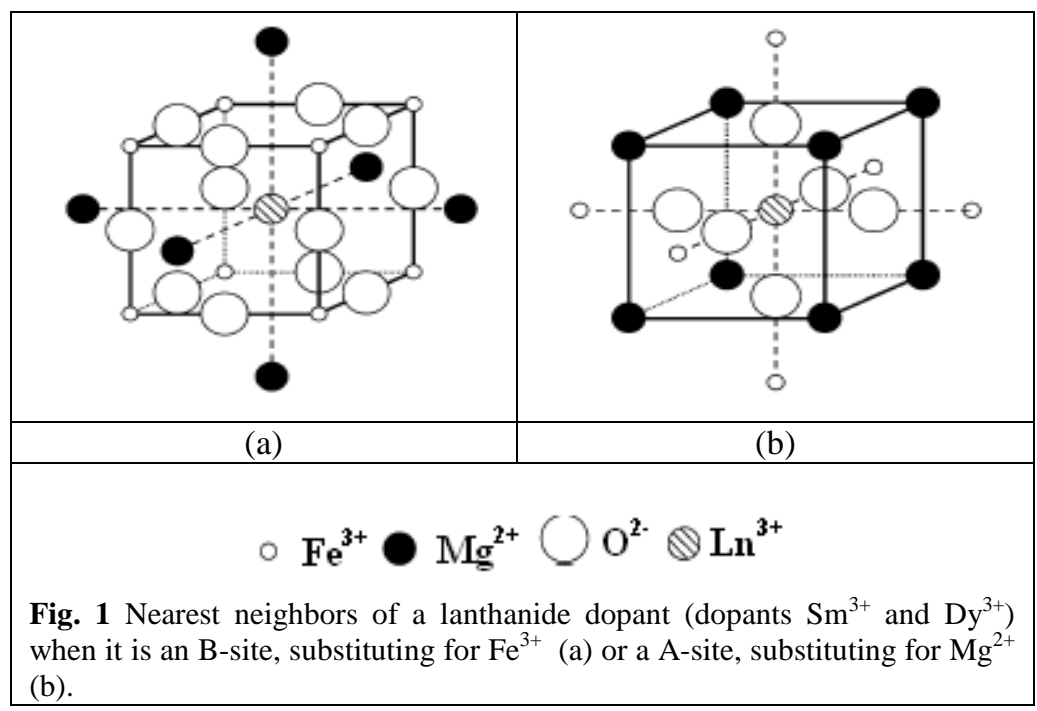

\begin{tabular}{|c|c|c|c|c|c|}
\hline \multicolumn{6}{|c|}{ TABLE 1: A-Site Calculation Data, for $\left.a_{0}\right) 8.462 \AA$} \\
\hline$k$ & atom & $\mathbf{r}_{k}$ & $\mathbf{Z}^{k}$ & $C 4, k\left(1 / r_{k}^{5}\right) Z^{k}(\AA-5)$ & $C 6, k\left(1 / r_{k}{ }^{7}\right) Z^{k}(\AA-7)$ \\
\hline 1 & $\mathrm{O}^{2-}$ & $\sqrt{2 /}(2) a_{0}$ & -2 & $5.716 \times 10^{-5}$ & $1.11 \times 10^{-6}$ \\
\hline 2 & $\mathrm{Fe}^{3+}$ & $\sqrt{3 /}(2) a_{0}$ & +3 & $5.511 \times 10^{-5}$ & $8.32 \times 10^{-7}$ \\
\hline 3 & $\mathrm{Mg}^{2+}$ & $a_{0}$ & +2 & $2.016 \times 10^{-5}$ & $3.02 \times 10^{-8}$ \\
\hline
\end{tabular}

\begin{tabular}{|c|c|c|c|c|c|}
\hline \multicolumn{6}{|c|}{ TABLE 2: B-Site Calculation Data, for $\left.a_{0}\right) 8.462 \AA$} \\
\hline $\boldsymbol{k}$ & atom & $\mathbf{r}_{k}$ & $\mathbf{Z}^{k}$ & $C 4, k\left(1 / r_{k}^{5}\right) Z^{k}(\AA-5)$ & $C 6, k\left(1 / r_{k}{ }^{7}\right) Z^{k}(\AA-7)$ \\
\hline 1 & $\mathrm{O}^{2-}$ & $(1 / 2) a_{0}$ & -2 & $-6.452 \times 10^{-4}$ & $3.86 \times 10^{-6}$ \\
\hline 2 & $\mathrm{Mg}^{2+}$ & $(\sqrt{\mathbf{3} / 2}) a_{0}$ & +2 & $-8.709 \times 10^{-7}$ & $1.04 \times 10^{-8}$ \\
\hline 3 & $\mathrm{Fe}^{3+}$ & $a_{0}$ & +3 & $7.156 \times 10^{-7}$ & $2.47 \times 10^{-10}$ \\
\hline
\end{tabular}

For our $\mathrm{MgFe}_{2} \mathrm{O}_{4}$ calculations, we set the lattice constant, $\mathrm{a}_{0}$, equal to $8.462 \AA$ [9] Although $\mathrm{MgFe}_{2} \mathrm{O}_{4}$ is rhombohedral at $10 \mathrm{~K}$ (the EPR measurement temperature), the ion displacement relative to the cubic prototype is quite small.[10] Consequently, the use of cubic symmetry is an appropriate approximation.

$$
\begin{aligned}
& b_{4}=\sum b_{4, k}=k_{4} \sum C_{4, k} \frac{1}{r_{k}{ }^{5}} Z^{k} \\
& K_{4}=-F_{4}\left(r^{4}\right) a_{4} \frac{e^{2}}{4 \pi \epsilon_{0}} \\
& b_{6}=\sum b_{6, k}=k_{6} \sum C_{6, k} \frac{1}{r_{k}{ }^{7}} Z^{k} \\
& K_{6}=-F_{6}\left(r^{6}\right) a_{6} \frac{e^{2}}{4 \pi \epsilon_{0}}
\end{aligned}
$$

The simulations were performed to obtain the following parameters by using $\mathrm{C}++$ Programme.

$$
\begin{aligned}
& \sum C_{4, k} \frac{1}{r_{k}{ }^{5}} Z^{k} \\
& \sum C_{6, k} \frac{1}{r_{k}{ }^{7}} Z^{k}
\end{aligned}
$$

This program evaluates the spherical harmonics for each particular shell around either type of site. Fig. 1 shows the first three coordination spheres around a lanthanide in A- and Bsites, respectively. Table 1 shows the shell data for the first three fourth- and sixth order terms for A-site substitution as the parameter values were substituted in Eq(5), and Table 2 shows the same for B-site substitution for the vales obtained by substituting in $\mathrm{Eq}(6)$.. 


\section{THEORETICAL CONSIDERATIONS OF SITE OCCUPANCY \\ 3.1 The Valence of the Dopant}

The valence of the rare earth dopants is influenced by the surroundings. While most of the rare earths are rather stable as $3^{+}$ions, some of them can assume either $2^{+}$or $4^{+}$valence states as well. For the less stable ions, the following parameters are to be considered:

-The site: A-site drives the rare-earth ion towards lower valence, while B-site drives it towards higher valence. This is due to both columbic and strain energy contributions.

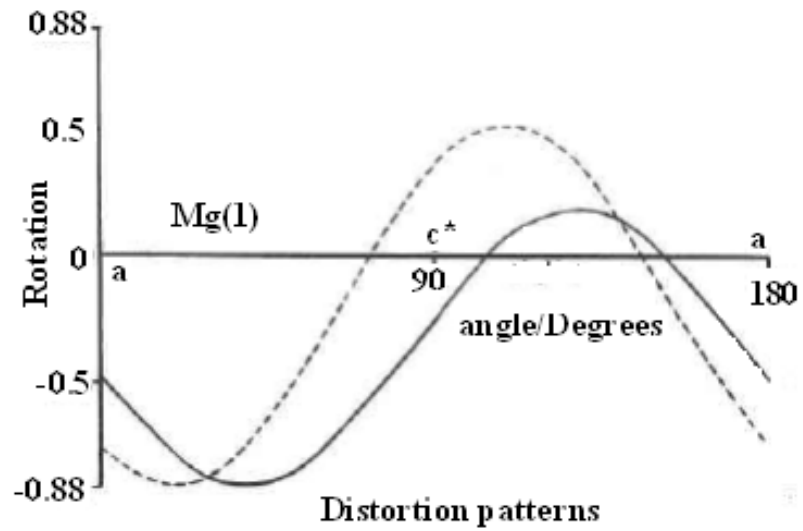

- Thermal energy: Higher temperature drives the ions towards higher valence.

- Electron/hole concentration (and oxygen partial pressure): For a given temperature,

Since the higher oxygen partial pressure give rise to higher concentration of holes and lower concentration of electrons. This drives the ions towards higher valiancy. It is already explained by [10] that the impurities choose their sites according to the local conditions during the incorporation. These impurities would not be expected to change their site at low temperatures.

Fig. 2. Comparison of the zero field splitting pattern of the most intense spectrum of $\mathrm{Mg} 2 *$ for rotation around the $\mathrm{b}$ axis (solid lines) with the distortion pattems of the four sites of divalent cations calculated from the crystal structure data (dashed lines). Both quantities are drawn as dimensionless distortions (:effective fractions of a ligand at normal bond distance; see Heming and Lehmann, 1987). Clearly the ZFS pattern is almost equally compatible with occupation of the Mgl and Mg3, sites.

\begin{tabular}{|c|c|c|c|c|c|c|c|c|c|}
\hline Ion & $\begin{array}{l}\mathbf{k 4} \\
\left(\mathrm{cm}^{-1} \AA^{5}\right)\end{array}$ & $\begin{array}{l}\mathbf{k 6} \\
\left(\mathrm{cm}^{-1} \AA^{7}\right)\end{array}$ & site & $\begin{array}{l}\text { b4 } \\
\left(\mathrm{cm}^{-1}\right)\end{array}$ & $\begin{array}{l}\text { b6 } \\
\left(\mathrm{cm}^{-1}\right)\end{array}$ & $\mathbf{X}$ & W & $\begin{array}{l}\text { ground } \\
\text { state }\end{array}$ & g value \\
\hline \multirow[t]{2}{*}{$\mathrm{Sm}^{3+}$} & -2593 & 0 & A & -0.827 & 0 & -1 & + & Г8 & \\
\hline & & & $\mathrm{B}$ & 71.3 & 0 & -1 & + & 「7 & 1.429 \\
\hline \multirow[t]{2}{*}{$D y^{3+}$} & 42.77 & -186.7 & A & 0.0136 & -0.0531 & -0.2 & - & I8(3) & \\
\hline & & & B & -1.176 & 0.126 & -0.9 & + & І6 & 6.667 \\
\hline
\end{tabular}

Table 3: Calculated Results for Non-S-State Lanthanides: K4, K6, b4, b6, x, and W values, predicted ground state, and $\mathrm{g}$ values

However, changes in valence depending on temperature and oxygen partial pressure may occur while the cation sublattice is in a meta stable state. Lea, Leask, and Wolf (LLW) performed calculations that detail the $\Gamma_{i}$ energy levels for possible ratios of the fourth- and sixth-order coefficients [11] The occupancy of Ml, M3 (Mg), sites are shown in Fig. Ml has a larger average bond distance than M3, it should be the one that is preferentially occupied, according to the principle of size mismatch. A possible cause for the low occupancy of the M2 site (despite its having even larger average bond distance than $\mathrm{Ml}$ ) may be the variation of the individual $\mathrm{O}$ bond lengths for this site, although they are not very large (0.09 and $0.02 \AA$ for : M1 andM3, respectively). 


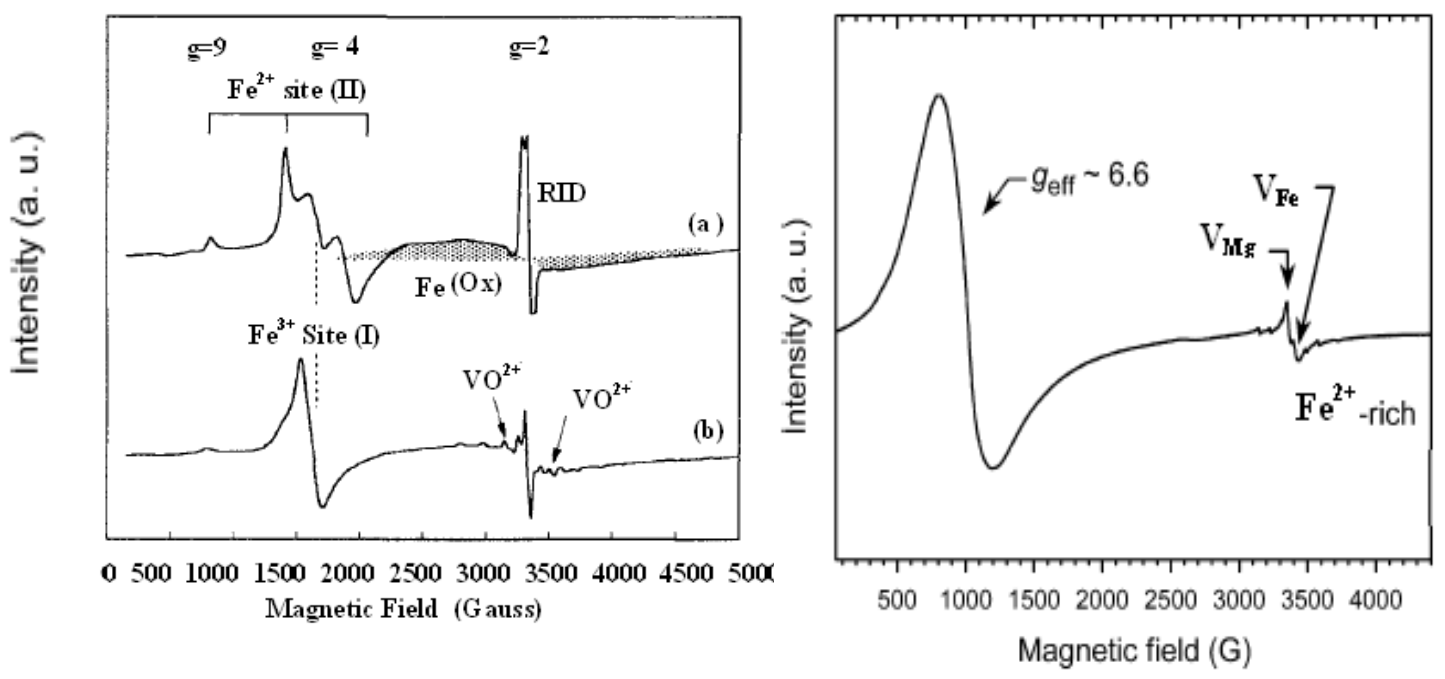

Fig.3. EPR spectra of Dy-Sm doped Magnesium ferrite

\section{RESULTS AND DISCUSSION 4.1 $\mathrm{Sm}^{3+-}$ Doping}

The electronic configuration of $\mathrm{Sm}^{3+}$ is $4 \mathrm{f}^{5}$ with a free-ion ground state of $6 \mathrm{H} 5 / 2$. The cubic crystal field splitting is the same as that for $\mathrm{Ce}^{3+.}$ Our calculations (Table 3 ) predict that $\mathrm{A}$ site $\mathrm{Sm}^{3+}$ has a $; 8$ ground state, whereas $\mathrm{B}$ site $\mathrm{Sm}^{3+}$ has a ground state of $; 6$, wi th a $g$ value of 1.429 . Experimentally, no signal was observed for $\mathrm{Sm}^{3+}$ in either $\mathrm{Mg}$-rich or Fe-rich samples, though magnetic field scans were performed to field levels equivalent to $g$ values as low as $1 / 2$. This suggests either that, at the EPR measurement temperature of $10 \mathrm{~K}$, the $\mathrm{Sm}$ in $\mathrm{MgFe}_{2} \mathrm{O}_{4}$ is largely $\mathrm{Sm}^{2+}$ or that the signal from the $\mathrm{Sm}^{3+}$ is too broad to be detected. Though $\mathrm{Sm}^{3+}$ has not previously been studied in $\mathrm{MgFe}_{2} \mathrm{O}_{4}$ by EPR, it has been studied by luminescence and found to be amphoteric, occupying both A- and B-sites in $\mathrm{MgFe}_{2} \mathrm{O}_{4}$ [13]. Furthermore, $\mathrm{Sm}^{3+}$ at the $\mathrm{Fe}^{2+}$ site was found to have a cubic crystal field regardless of the $\mathrm{MgFe}_{2} \mathrm{O}_{4}$ crystal symmetry (e.g., rhombohedral, orthorhombic, tetragonal, or cubic)[14].

$\mathrm{Sm}$ is known to be a $2^{+}$as well as a $3^{+}$ion. At high temperatures it is $3^{+}$.The experimental data suggest that at room temperature it is also $3^{+}$. This is in accordance with the room temperature conductivity measurements of Rotenberg et al. [15]. The lack of signal in the EPR measurements suggest that $\mathrm{Sm}^{3+}$ cation it is on the A-site.

\section{2 $\mathrm{Dy}^{3+-}$ Doping}

We now return to our discussion of non-S-state Kramers ions, continuing with $\mathrm{Dy}^{3+}$ whose electronic configuration is $4 \mathrm{f9}$, with a free-ion ground state of $6 \mathrm{H}_{15 / 2}$. Our point charge calculation predicts $\mathrm{I}_{8}$ for $\mathrm{Dy}^{3+}$ in the A-site and $\mathrm{I}_{6}$ for the Bsite $\left(g \Gamma_{6}\right)$ 6.667) ground states (see Table 3 ). $\mathrm{Dy}^{3+}$ has not, to our knowledge, been observed in $\mathrm{MgFe}_{2} \mathrm{O}_{4}$ before by EPR. The EPR spectra of Dy-doped Mg- and Fe-rich Dysprosium Samarium are shown in Fig 3. A large, broad, anisotropic peak, with $g 6.6$ due to $\mathrm{Dy}^{3+}$ is visible. No hyperfine lines from ${ }^{161}$ Dy and ${ }^{163}$ Dy are visible, most likely because of the broadness of the $\mathrm{Dy}^{3+}$ signal relative to the expected hyperfine coupling constants[16] The $g$ value of $\mathrm{Dy}^{3+}$ observed in $\mathrm{Mg}$ rich $\mathrm{MgFe}_{2} \mathrm{O}_{4}$ corresponds to $\mathrm{Dy}^{3+}$ in the $\mathrm{B}$ site. However, no signal from the A-site $\mathrm{Dy}^{3+}$ is apparent.
The intensity of the $\mathrm{Dy}^{3+}$ is strongly dependent upon the $\mathrm{Mg} /$ Fe ratio, with the Mg-rich sample showing 3 times the $\mathrm{Dy}^{3+}$ signal as compared to the Fe-rich (Fig.3 EPR spectra). The decrease in $\mathrm{Dy}^{3+}$ intensity as the Fe concentration increases matches what one would expect for a B site dopant. A-site $\mathrm{Dy}^{3+}$ could be reduced to $\mathrm{Dy}^{2+}$ at low temperature by capture of an electron, thus making it invisible to EPR. The higher $V$ Fe signal in the Fe-rich sample demonstrates the need for acceptors and suggests that during the incorporation, at high temperature, the Dy valence is $3+$ on the A-site also.

\section{CONCLUSION}

Dysprosium, Samarium doped magnesium ferrites were investigated using EPR studies, for determining the site occupancy of these lanthanides (Dy-Sm). In this investigation we found that $\mathrm{Dy}^{3+}$ was found to be a B-site dopant because of its signal intensity in EPR spectra $\mathrm{Dy}^{3+}$ is an amphoteric dopant in $\mathrm{MgFe}_{2} \mathrm{O}_{4}$ and its solubility limit is higher on the Bsite. For $\mathrm{Sm}^{2+}$ dopant, lack of signals were obtained from EPR spectra. The experimentally observed doping behavior of $\mathrm{Dy}^{3+}$ $-\mathrm{Sm}^{3+}$ in $\mathrm{MgFe}_{2} \mathrm{O}_{4}$ suggests that as a $\mathrm{Sm}^{3+}$ cation it is on the A-site. The determination of Dopant site will be helpful for the analytical studies of permittivity and permeability to design Microstrip patch antenna to be used in pervasive computing.

\section{REFERENCES}

[1] Hawthorne, F C (1983) Quantitative characterization of site-occupancies in minerals. American Mineralogist, 68, $287-306$.

[2] Vasant Naidu et.al Designing a dual ISM band implantable antenna for medical monitoring applications using Dy- Sm doped magnesium Nano Ferrite material International Journal of Computer Applications (0975 8887), Vol 44, No 12, Page 1-4.

[3] Lee, W. H.; Groen, W. A.; Schreinemacher, H.; Hennings, D. J. Electroceram. 2000, 5, 31.

[4] Tsur, Y.; Randall, C. A. AIP Conf. Proc. 2000, 535, 283.

[5] Hennings, D. F. K. J. Eur. Ceram. Soc. 2001, 21, 1637. 
[6] Timothy D. Dunbar, "Electron Paramagnetic Resonance Investigations of Lanthanide-Doped Barium Titanate:Dopant Site Occupancy” J. Phys. Chem. B 108 (2004) 908-917.

[7] Abragam, A.; Bleaney, B. Electron Paramagnetic Resonance of Transition Ions; Clarendon Press: Oxford, U.K., 1970.

[8] Bureau, B.; Silly, G.; Buzare, J. Y. J. Phys. Chem. Solids 1998, 59, 951.

[9] Siegel, E.; Mu“ller, K. A. Phys. ReV. B 1979, 20, 3587.

[10] Tsur, Y.; Dunbar, T. D.; Randall, C. A. J. Electroceram. $2001,7,25$

[11] Acr,.qla B. Vassrr,rrou-Dova, EPR-determined site distributions of low concentrations of transition-metal ions in minerals: Review and predictions, American Mineralogist, Volume 78, i993, pages 49-55.

[12] Lea, K. R.; Leask, M. J. M.; Wolf, W. P. J. Phys. Chem. Solids 1962, 23, 1381.

[13] Makishima, S.; Hasegawa, K.; Shionoya, S. J. Phys. Chem. Solids 1962, 23, 749 .

[14] Makishima, S.; Yamamoto, H.; Tomotsu, T.; Shionoya, S. J. Phys. Soc. Jpn. 1965, 20, 2147.

[15] B.A. Rotenberg, Yu. L. Danilyuk, E.I. Gindin, and V.G. Prokhvatilov, Soviet Phys.-Solid State, 7, 2465 (1966).

[16] Reynolds, R. W.; Boatner, L. A.; Chen, Y.; Abraham, M. M. J.Chem. Phys. 1974, 60, 1593. 